\title{
Evidence for high saturation of porous amorphous carbon films by noble gases
}

\author{
N.V. Krainyukova \\ B. Verkin Institute for Low Temperature Physics and Engineering of the National Academy of Sciences of Ukraine \\ 47 Lenin Ave., Kharkov 61103, Ukraine \\ E-mail: krainyukova@ilt.kharkov.ua \\ Received January 26, 2009
}

\begin{abstract}
Results of the electron diffraction study of $\mathrm{Kr}$ and Xe deposits on amorphous porous carbon substrates are reported. We have observed mixtures of crystallites typical of condensates formed at such substrates at low deposition temperatures. However, in the warming process at temperatures about three-to-five degrees below the sublimation point characteristic of flat substrates, the diffraction patterns demonstrate that large crystallites gradually disappear and a highly disordered matter forms. Such transformed samples are kept inside substrates several dozens degrees above the sublimation points which are typical of these substances at flat (e.g. metallic) substrates. We ascribe these features to specificity of composites formed from noble gases strongly bonded inside porous carbon matrices due to capillary filling at temperatures close to the sublimation points.
\end{abstract}

PACS: 61.14. $-\mathbf{x}$ Electron diffraction and scattering; 61.46.-w Nanoscale materials ; 81.05.Rm Porous materials; granular materials; 81.05.Uw Carbon, diamond, graphite.

Keywords: 7carbon nanopores, inert gases, absorption, electron diffraction.

\section{Introduction}

In early 90 s, in parallel with discovery and first extensive investigations of fullerenes and carbon nanotubes, several scientific groups found that side-products in relevant production processes (i.e. in carbon-arc synthesis and in carbon nanotubes growth) also possess some intriguing and unique properties. Such materials were shown to be similar to so-called random Schwarzite forms which display interconnected net of channels between negatively-curved graphitic sheets [1-6]. These forms comprise pentagons, hexagons, heptagons and higher order rings of $s p^{2}$-coordinated carbon atoms, which was confirmed, in particular, in studies of radial distribution functions in diffraction experiments [4-6]. Carbon Schwarzites were found to be more stable than fullerenes. Size of free spaces between sheets (or «pores» of arbitrary forms) stretch from several angstroms in arc discharge experiments to microns in other techniques applying metal catalysts [3]. Investigations revealed the unique similarity between random Schwarzite forms and films of «amorphous» carbon [1] widely used in electron microscopy and electron diffraction experiments.

Last years many experimental groups carry out investigations of fullerenes and nanotubes saturation by different gaseous admixtures and study various aspects of relevant processes [7-12]. One of the most important tasks connected with potential applications is to attain as high saturation as possible, which, additionally, would be stable at elevated temperatures. For fullerites, the basic restriction in these processes is connected with the fact that, as a rule, only octahedral interstitial sites are available for gaseous admixtures. Therefore, even at 100\% filling, the upper limit in such saturation is the ratio one admixture atom or molecule per one fullerene molecule, i.e. 1/60 or $1 / 70$ with respect to atomic count. One encounters similar limitation in the saturation of carbon nanotubes of small diameters.

In this work we study saturation of amorphous porous carbon films by noble gases: $\mathrm{Kr}$ and Xe. Such matrices are attractive for the saturation study due to small dimensions of pores (which can be filled by gaseous admixtures with 
strong bonding with pore walls) and to the absence of huge spaces (like in fullerene molecules) which are not accessible for the penetration by gas atoms and molecules.

\section{Experimental technique and the analysis method}

We report the results of the high-energy $(50 \mathrm{keV})$ electron diffraction study of $\mathrm{Kr}$ and $\mathrm{Xe}$ deposits on amorphous porous carbon substrates. Porous carbon films were produced from vacuum-sublimated graphite by the electric current heating of thin carbon rods; the arc discharge with sparkling evaporation of large pieces of graphite was avoided. Substrates were put onto a copper net (of high thermal conductivity and transparent for electrons) by means of floating. Prepared in such a way films were heated to $50-60^{\circ} \mathrm{C}$ in high vacuum and then kept inside vacuum chamber for 2-3 days before the low-temperature experiments. Gaseous krypton and xenon were deposited on amorphous carbon substrate inside the electron diffraction set-up at 23 and $40 \mathrm{~K}$, respectively. In parallel, we made a similar deposition onto an aluminum film which was placed on the same sample holder inside the helium cryostat. Produced in such a way samples were gradually warmed up, step by step, to temperatures far above the sublimation points ( $64 \mathrm{~K}$ for Xe and $44 \mathrm{~K}$ for $\mathrm{Kr}$ ) typical of studied substances in our specific vacuum conditions. The diffraction data were recorded in the form of numerical files. The effective thickness of deposits was about 150-200 $\AA$ and characterized the total amount of condensed materials.

Diffractograms were analyzed applying the simulations which were similar to those described previously [6, 17-22], i.e. basing on the assumption of a wide variety of possible structures and sizes involved in the formation of observed diffraction patterns. Single crystals, such as cuboctahedra, and hcp (hexagonal close-packed) clusters were included in our analysis together with multiply-twinned particles (MTPs) with five-fold symmetry, i.e. non-crystallographic multi-shell icosahedra (ico) [13] and Mark's decahedra (dec) [14], with, initially, from one up to twenty shells for every structural type. Such MTPs are known [15-17] to appear in open and partly-open geometry due to their energetic preference as compared with fcc (face centered cubic) and hcp clusters. The diffracted intensity (per atom) for each cluster $k$ comprising $N_{k}$ atoms was calculated using Debye formula:

$$
\begin{aligned}
I_{\text {calc }, k}(s)=\left(f^{2}+s^{-4}\right. & S(s)) /(1-t)+\left(2 f^{2} / N_{k}\right) \exp \left[-<u^{2}>_{k} s^{2}\right] \times \\
& \times\left[\Sigma\left(\sin s r_{i j}\right) /\left(s r_{i j}\right)\right]_{k} .
\end{aligned}
$$

Here the fraction $t$ of atoms is assumed to be free (i.e. not bonded to the cluster or highly disordered). The summation in the interference function runs in the cluster $k$ over all pairs of atoms $i>j$, separated by the distance $r_{i j}$; the
Debye-Waller factor $\left.\exp \left[-<u^{2}\right\rangle_{k} s^{2}\right]$ takes into account the mean-square atomic displacements $\left\langle u^{2}\right\rangle_{k}, f(s)$ is the elastic atomic scattering factor for electrons, $S(s)$ is the incoherent $\mathrm{x}$-ray scattering factor. The superposition of diffracted intensities $I_{\text {calc }}$ from different clusters $k$ with their relative weights $w_{k}$, i.e. $I_{\text {calc }}=\Sigma w_{k} I_{\text {calc }, k}$, was compared with experimental intensities $I_{\text {exp }}$ using the reliability factor $R$ in the form

$$
R=\Sigma\left|I_{\text {exp }}-I_{\text {calc }}\right| / \Sigma\left(I_{\text {exp }}+I_{\text {calc }}\right) ;
$$

the summation was performed along the experimental and calculated diffraction patterns with the equidistant step $\sim 0.015 \AA^{-1}$ in $s=4 \pi \sin (\theta) / \lambda$ ( $\theta$ is the diffraction angle and $\lambda$ is the wavelength of electrons). The values $w_{k}$ were involved in the total analysis as variables in the $R$-factor minimization procedure (total $\Sigma w_{k}=1$ ).

\section{Results and discussion}

Both objects under study ( $\mathrm{Kr}$ and $\mathrm{Xe}$ deposits) passed through very similar transformations. Evolution of diffraction patterns is presented in Fig. 1 and Fig. 2 for Xe and $\mathrm{Kr}$, respectively.

Samples were prepared at low temperatures and then warmed up. We kept the samples at temperatures a few degrees below the sublimation points, i.e. at $59 \mathrm{~K}$ for $\mathrm{Xe}$ and at $41.5 \mathrm{~K}$ for $\mathrm{Kr}$. We observed that distinct diffraction peaks gradually disappear and strong halos centered approximately in the position of the most intensive peak (111) of the crystalline fcc phases for both objects became visible (Figs. $1, b$ and $2, b$ ). These halos evolved in time and upon warming, but at temperatures several dozens degrees above the sublimation points they were still visible. No peaks or other signs of the presence of deposited samples were observed for the metallic substrates above the sublimation points.

We can specify several important stages in transformations of samples prepared onto amorphous carbon substrates. The distinct halos formed below the sublimation points; latter no essential transformations occurred, either in time or at warming, until the characteristic sublimation points were reached, except for only some small changes in the form of the background (Fig. 1,c). At $64 \mathrm{~K}$ for Xe and at $44 \mathrm{~K}$ for $\mathrm{Kr}$ (the clearly specified sublimation points), the intensity of main halos essentially diminished but halos were still preserved with the high enough peak intensity. The maximum intensity $I_{\max }$ of the halo's peak and its evolution with the temperature for $\mathrm{Xe}$ and $\mathrm{Kr}$ are shown in Fig. 3 and Fig. 4, respectively. The specific features described above and also some smaller transformations are distinctly visible.

The analysis method, described in the previous section, was applied in order to determine the structure of deposits. We obtained the size and structure distribution functions (Figs. 5,d-f) corresponding to the different 


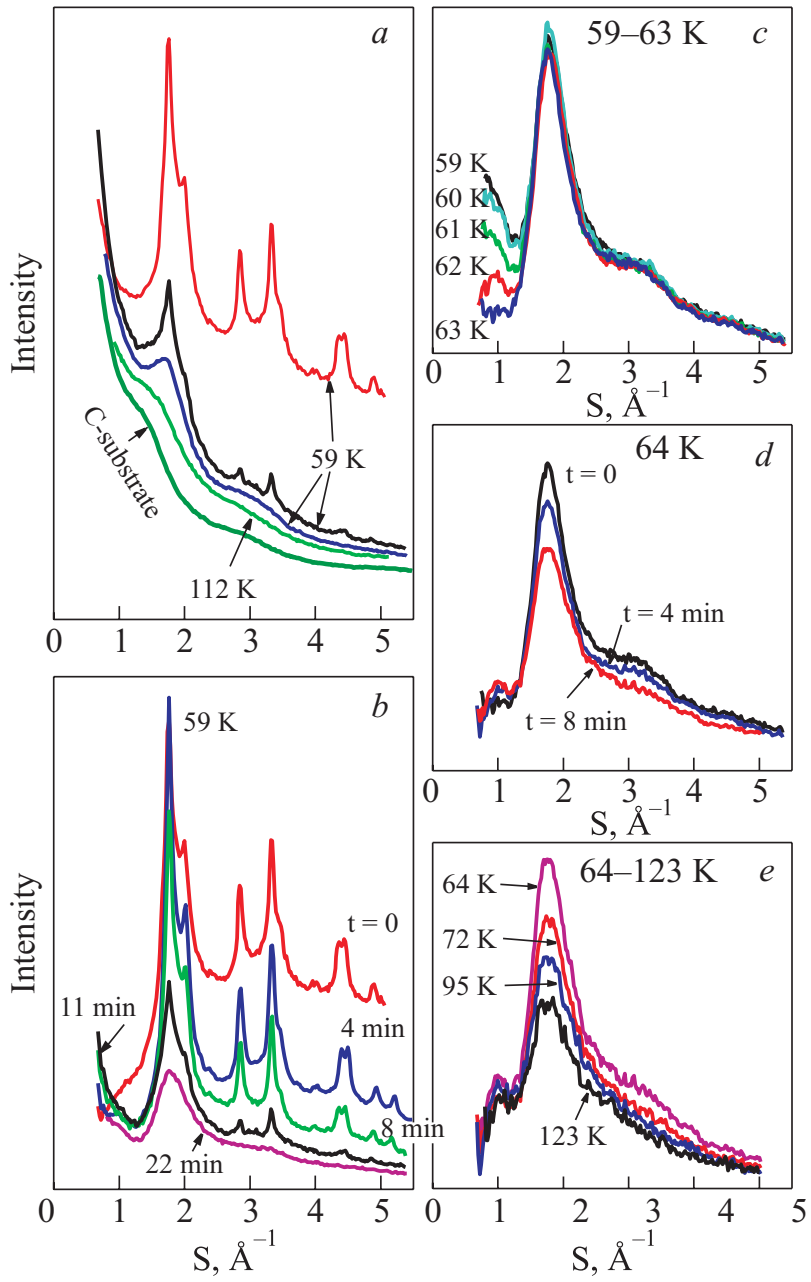

Fig. 1. (Color online) Temperature and time $(t)$ evolution of diffraction patterns from Xe films deposited on amorphous carbon substrates marked in the Figure. In the $(b)-(e)$ subfigures we show diffraction patterns with the carbon substrate contribution subtracted. The time evolution was observed only at 59 and $64 \mathrm{~K}$, at other temperatures no noticeable time-dependent changes were found.

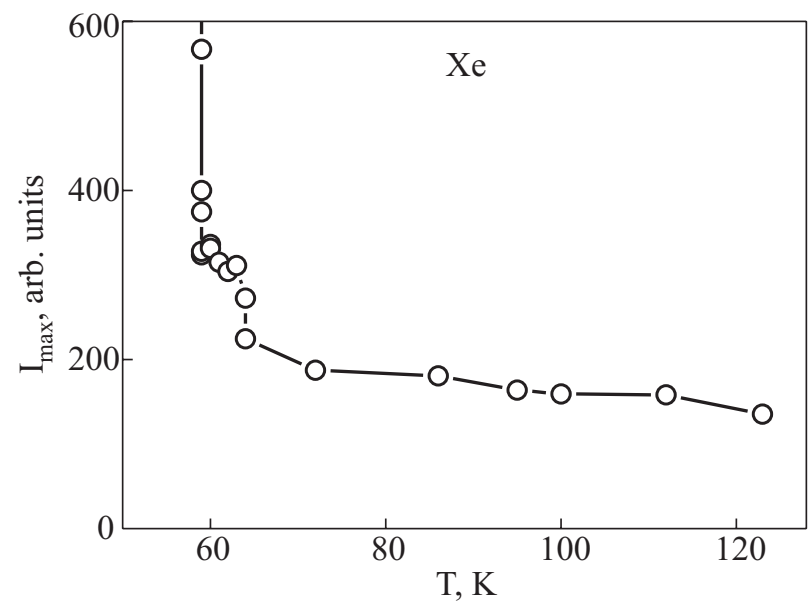

Fig. 3. Temperature dependence of $I_{\max }$ for Xe samples prepared on amorphous carbon substrate.
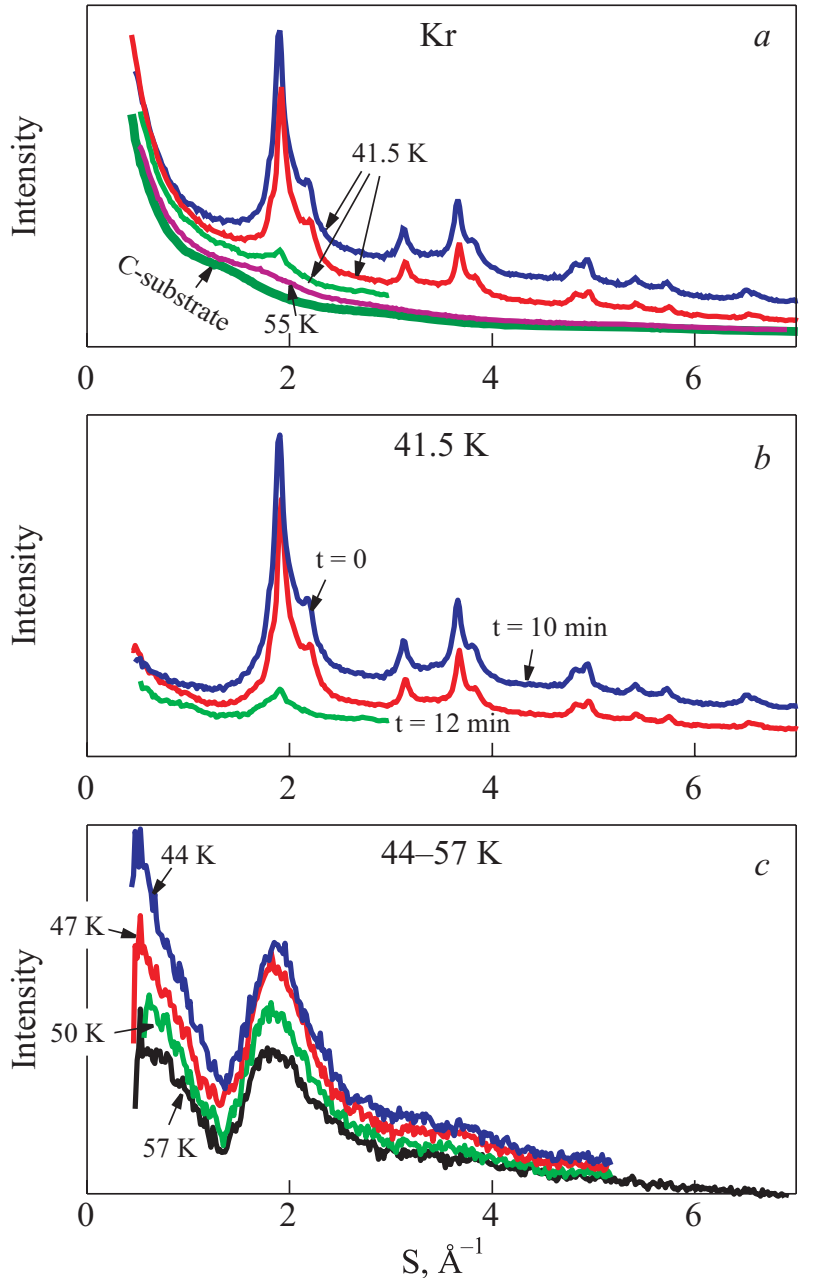

Fig. 2. (Color online) Temperature $(a, c)$ and time $(a, b)$ evolution of diffraction patterns from $\mathrm{Kr}$ films deposited on amorphous carbon substrates marked in the Figure. In the $(b)$ and (e) subfigures we show diffraction patterns with the carbon substrate contribution subtracted. The time evolution was observed only at 41.5 and $44 \mathrm{~K}$, at other temperatures no noticeable time-dependent changes were found.

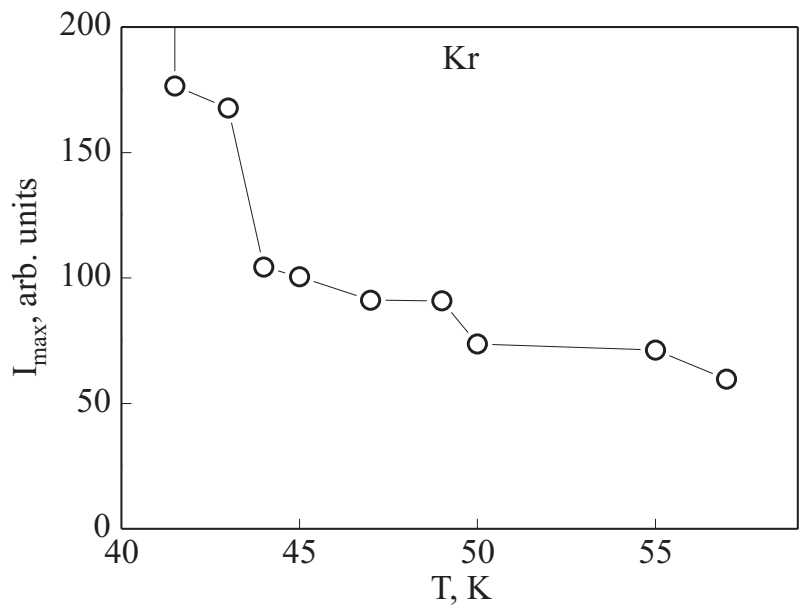

Fig. 4. Temperature dependence of $I_{\max }$ for $\mathrm{Kr}$ samples prepared on amorphous carbon substrate. 

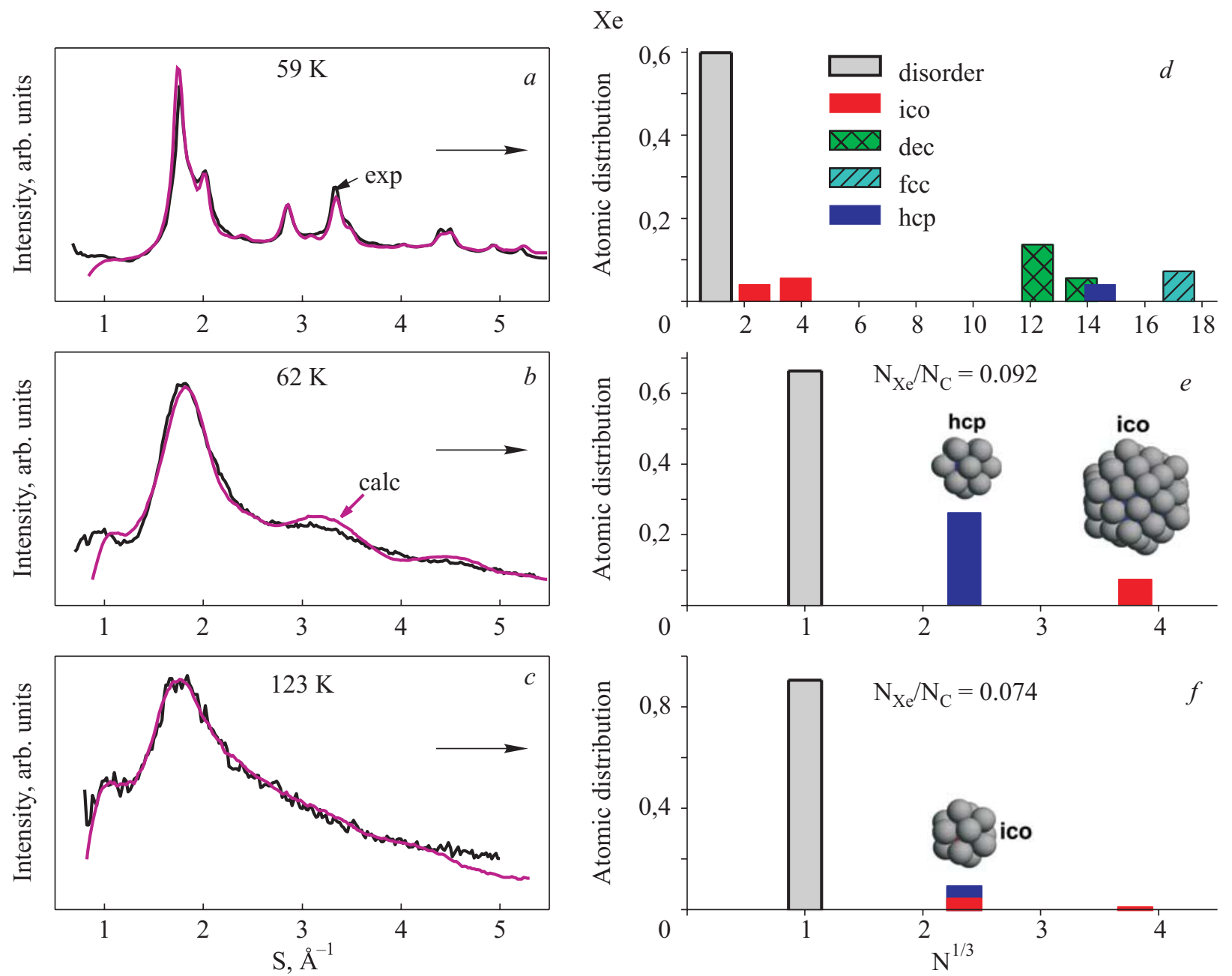

Fig. 5. (Color online) Experimental (black) and calculated (gray, color) diffractograms for Xe samples (left) at earlier stages of transformation $(a)$, after the distinct halo formed $(b)$ and at $123 \mathrm{~K}$, i.e. $\sim 60 \mathrm{~K}$ above the sublimation point for Xe. The relevant distribution functions are shown on the right. Cluster identifications in the inset and the structures of smallest clusters are shown.

stages of transformations. At the first stage, when a large group of diffraction peaks were observed (Fig. 5,a), the relevant distribution (Fig. 5,d) demonstrates that microsized ( $N<60$ atoms) clusters are mixed with much larger meso-sized species $(N>1000$ atoms) (for classification see [3]). The noticeable gap between smallest clusters $N \sim 13-60$ atoms and $N \sim 700-1000$ atoms and abundance of MTPs, i.e. clusters with five-fold symmetry are evident. The essential contribution of the part $t$ of deposits, which is not bonded to clusters, is presented at all stages and positioned in the distributions at $N=1$.

The next distribution (Fig. 5,e) corresponds to the stage (Fig. 5,b) when the distinct halo (formed at $59 \mathrm{~K}$ ) is kept nearly unchanged up to the sublimation point at $64 \mathrm{~K}$. In this case, the distribution shows that a large population of meso-sized and macro-sized clusters with $N>1000$ atoms disappeared and only the smallest clusters with $N=13$ and $N \sim 60$ atoms as well as the disordered fraction are still presented in the distribution. Evi- dently, large clusters partly sublimate and/or penetrate inside porous amorphous carbon matrix at temperatures slightly below the sublimation points, which is confirmed by the presence of the strong halo. The next distribution shown in Fig. 5, $f$ demonstrates that clusters with $N \sim 60$ atoms also disappear at elevated temperatures and only two kinds of the matter are kept inside the porous matrix, i.e. smallest clusters with $N \sim 13$ atoms and the disordered fraction $t$. The halo is preserved several dozens degrees above the sublimation points.

According to our observations, we conclude that the substance directly deposited on substrate at low preparation temperature forms large clusters, which later penetrate in the carbon matrix at temperatures slightly below the sublimation points. We explain this effect by thermally activated processes that make it possible to fill smallest pores due to diffusion. Thus, large clusters form in the essentially open geometry, which explains the wide application of these so-called «amorphous» carbon films 
in the electron microscopy and the electron diffraction as supporting substrates. But it was shown also, and has been confirmed in our previous studies [6,18-22] that carbon films prepared by means of vacuum sublimation of graphite can be characterized as a porous matrix with very fine channels. This finding correlates with the essentially damped atomic dynamics in neon clusters formed in pores [19], which is ascribed to strong interaction of neon atoms with carbon walls.

Our analysis enabled us also to estimate the degree of saturation of our carbon matrices by noble gases, applying the Debye formula and recording the carbon contribution to the total picture before deposition of samples. These estimations are included in the Figs. 5, e, $f$ as ratios of noble gases atoms to carbon atoms participating in the formation of the diffraction patterns. In the case of Xe at $59 \mathrm{~K}<T<64 \mathrm{~K}$ this ratio is $N_{\mathrm{Xe}} / N_{\mathrm{C}} \sim 9 \%$ and at $123 \mathrm{~K}$ $N_{\mathrm{Xe}} / N_{\mathrm{C}} \sim 7 \%$. For $\mathrm{Kr}$ this ratio is also about few percents. The detailed analysis of the most optimal conditions for gas diffusion and penetration into carbon matrices will be important in the future; here we can conclude that saturations attained in our experiments are certainly much higher than it can be expected for fullerites and carbon nanotubes of small diameters.

The important question is, how noble gases are distributed inside porous carbon matrices. Exact answers can be obtained by direct modeling such composites applying (e.g.) the Monte Carlo method $[23,24]$. In particular, the theory predicts that Xe atoms tend to occupy the center of a graphite hexagon [23] and also always try to accommodate the substrate structure. Simple estimations show that in our experiments we should expect maximal saturations about or lower than $17 \%$, which agrees with the experimental values.

The concept of adsorption, wetting and capillary condensation was well established for van der Waals liquids in porous matrices [25-27], and below the triple point we could expect the reduced wetting for substances which were studied in this work, because of substrate roughness [26] or elastic compression [25]. However, our results evince that solid $\mathrm{Kr}$ and $\mathrm{Xe}$ condensates formed in pores closely to their sublimation points are stable in a wide temperature range, which correlates with the observation of a capillary condensation of solid Ar studied by means of x-ray diffraction [27].

\section{Conclusions}

Mixtures of xenon and krypton crystallites form on amorphous porous carbon substrates at low deposition temperatures. In the warming process, at temperatures which are about three-to-five degrees below the sublimation points characteristic of flat substrates, the evolution of the experimental diffraction patterns demonstrates that large crystallites gradually disappear and a highly disor- dered matter strongly bonded with carbon pore walls forms. Such transformed samples are kept inside carbon matrices at least several dozens degrees above the sublimation points typical for these substances at flat (e.g. metallic) substrates. In other words, our experiments demonstrate much stronger bonding of noble gases inside carbon porous matrices as compared with deposits on conventional surfaces. We ascribe these features to specificity of composites formed from noble gases inside porous matrices due to capillary condensation at temperatures close to the sublimation points. The structure of a formed matter is analyzed. Our estimations of saturations show that the ratio of noble gas atoms to carbon matrix atoms can attain, in studied objects, $\sim 10 \%$, which is definitely much higher that achieved with fullerites and carbon nanotubes. We expect that our results can be of general importance for the future studies of such systems as well as for filling and storage of numerous liquid and gaseous materials.

The author is grateful to V.G. Manzhelii, M.A. Strzhemechny, P. Leiderer, J.P. Toennies, M.I. Bagatskii, and A.I. Krivchikov for fruitful discussion of this work.

1. S.J. Townsend, T.J. Lenosky, D.A. Muller, C.S. Nichols, and V. Elser, Phys. Rev. Lett. 69, 921 (1992).

2. D. Vanderbilt and J. Tersoff, Phys. Rev. Lett. 68, 511 (1992).

3. E. Barborini, P. Piseri, P. Milani, G. Benedek, C. Ducati, and J. Robertson, Appl. Phys. Lett. 81, 3359 (2002).

4. F. Li and J.S. Lannin, Phys. Rev. Lett. 65, 1905 (1990).

5. D.R. McKenzie, D. Muller, and B.A. Pailthorpe, Phys. Rev. Lett. 67, 773 (1991).

6. N.V. Krainyukova, Surf. Interf. Anal. 38, 469 (2006).

7. A.N. Aleksandrovskii, V.G. Gavrilko, V.B. Esel'son, V.G. Manzhelii, B. Sundqvist, B.G. Udovidchenko, and V.P. Maletskiy, Fiz. Nizk. Temp. 27, 1401 (2001) [Low Temp. Phys. 27, 1033 (2001)].

8. A.N. Aleksandrovskii, A.S. Bakai, D. Cassidy, A.V. Dolbin, V.B. Esel'son, G.E. Gadd, V.G. Gavrilko, V.G. Manzhelii, S. Moricca, and B. Sundqvist, Fiz. Nizk. Temp. 31, 565 (2005) [Low Temp. Phys. 31, 429 (2005)].

9. V.G. Manzhelii, A.V. Dolbin, V.B. Esel'son, V.G. Gavrilko, G.E. Gadd, S. Moricca, D. Cassidy, and B. Sundqvist, Fiz. Nizk. Temp. 32, 913 (2006) [Low Temp. Phys. 32, 625 (2006)].

10. Yu.E. Stetsenko, I.V. Legchenkova, K.A. Yagotintsev, A.I. Prokhvatilov, and M.A. Strzhemechny, Fiz. Nizk. Temp. 29, 597 (2003) [Low Temp. Phys. 29, 445 (2003)].

11. K.A. Yagotintsev, M.A. Strzhemechny, Yu.E. Stetsenko, I.V. Legchenkova, and A.I. Prokhvatilov, Physica B381, 224 (2006).

12. I.V. Legchenkova, A.I. Prokhvatilov, Yu.E. Stetsenko, M.A. Strzhemechny, and K.A. Yagotintsev, Fiz. Nizk. Temp. 33, 119 (2007) [Low Temp. Phys. 33, 89 (2007)].

13. A.L. Mackay, Acta Cryst. 15, 916 (1962).

14. L.D. Marks, Philos. Mag. A49, 81 (1984).

15. N.V. Krainyukova, Thin Solid Films 515, 1658 (2006) (and references therein). 
16. N.V. Krainyukova, Eur. Phys. J. D43, 45 (2007).

17. V. Kiryukhin, E.P. Bernard, V.V. Khmelenko, R.E. Boltnev, N.V. Krainyukova, and D.M. Lee, Phys. Rev. Lett. 98, 195506 (2007).

18. N.V. Krainyukova and B.W. van de Waal, Thin Solid Films 459, 169 (2004).

19. N.V. Krainyukova, AIP Conf.Proc. 850, 390 (2006) [24th Int. Conf. on Low Temperature Physics, Orlando, FL (2005)].

20. N.V. Krainyukova, Thin Solid Films 515, 2792 (2006).

21. N.V. Krainyukova and V.G. Belan, Eur. Phys. J. D43, 169 (2007)
22. N.V. Krainyukova, Fiz. Nizk. Temp. 33, 747 (2007) [Low Temp. Phys. 33, 569 (2007)].

23. R. Etters, E. Flenner, B. Kuchta, L. Firlej, and W. Przydrozny, J. Low Temp. Phys. 122, 121 (2001).

24. L. Firlej, B. Kuchta, R. Etters, W. Przydrozny, and E. Flenner, J. Low Temp. Phys. 122, 171 (2001).

25. F.T. Gittes and M. Schick, Phys. Rev. B30, 209 (1984).

26. A. Esztermann, M. Heni, H. Löwen, J. Klier, M. Sohaili, and P. Leiderer, Phys. Rev. Lett. 88, 055702 (2002).

27. P. Huber and K. Knorr, Phys. Rev. B60, 12657 (1999). 